\title{
Recent progress of visible light nulling interferometry and first 1 million null result
}

\author{
Edouard Schmidtlin ${ }^{1}$, J. Kent Wallace ${ }^{1}$, Rocco Samuele ${ }^{2}$ \\ B. Martin Levine ${ }^{1}$, and Michael Shao ${ }^{1}$ \\ 1 Jet Propulsion Laboratory, California Institute of Technology, Pasadena, CA 91007 \\ 2 Northop Grumman Space Technology, Redondo Beach, CA 90278 \\ email: edouard.schmidtlin@jpl.nasa.gov
}

\begin{abstract}
In order to detect directly faint planets around other stars it is important to develop techniques to cancel the central starlight. We report progress in terms of null depth on the visible nulling experiments at JPL, essential for NASAs future space missions as well as for an upcoming sounding rocket based experiment. A level of 1 Million to 1 nulling ratio (1e-6 rejection ratio) has been obtained for the first time for visible laser light, and a level of 100000 to 1 nulling ratio has been obtained for a relatively large $(5 \%)$ bandpass at $650 \mathrm{~nm}$. Using a combination of a fiber array and deformable mirror a null of 1e-6 would correspond to a contrast of $1 \mathrm{e}-9$ at a distance of $2-3 \lambda$ in the airy disk plane, only $10 \mathrm{x}$ away from TPFs goal. The configuration used was a fiber fed Mach Zender type interferometer, using 2 or 3 mirrors in each two arm, placed in an enclosed air container. For broadband light we used tilted dispersion glass plates in the nuller arms and an avalanche photon diode module to cover the large dynamic range. We describe a variety of conditions that have to be met and optimized to reach very deep nulls and sub-nanometer optical path difference stability, such as optical alignment, symmetry in the two arms, mechanical stability and vibration isolation.
\end{abstract}

Keywords. extra solar planet detection, interferometry, nulling, optical path stabilization

\section{Introduction}

Since 1995 [1] about 150 exo-planets have been detected around other stars, using various methods, but a common fact is that these detections were indirect [2,3]. A few recent direct claims have been made but there is ambiguity, such as lack of proof of gravitational relationship, moreover the planets tend to be much larger than Earth types. It still remains a formidable challenge to observe an exoplanet directly, whether the light comes from the planet itself or is a reflection of light coming from the star. In order to detect and observe a faint planet near a bright star it is necessary to cancel the central starlight to high rejection ratios which leads to both a contrast and an angular resolution challenge. It is currently believed that such a challenge is out of the question from the ground because of atmospheric seeing and scatter, even with state of the art adaptive optics, so space observation is mandatory. In terms of contrast the planet to star ratio is $1 \mathrm{e}-9$ for a Jupiter and is $1 \mathrm{e}-10(\Delta \mathrm{mag}=25)$ for an Earth like planet in the visible. In the infrared the contrast is about 1000 times easier, but the search is more difficult. Assuming a theta at the 4th power dependency, a null of $1 \mathrm{e}-7$ would correspond to a contrast of $1 \mathrm{e}-10$ at a close distance of 1 airy spot $(1 \lambda / \mathrm{D})$.

A nulling interferometer (aka coronagraph) can consist of either a single large aperture telescope (with sheared pupil interferometer or equipped with a mask with two apertures), or an interferometric array of multiple telescopes [4]. In terms of resolution 
very large telescopes are not necessary: for an Earth at 10parcsec (0.1 $\operatorname{arcsec})$ D can be only $1.5 \mathrm{~m}$, whereas for a Jupiter $0.3 \mathrm{~m}$ will suffice. Whereas a classic Michelson interferometer with one of its mirror displaced by $\lambda / 2$ can achieve a null of no better than .0005 and .002 for a bandpass of respectively 5\% and 10\%, a dedicated Achromatic Nulling Interferometer is needed for deeper nulls over larger bandwidths of astronomical interest. Efforts have been conducted in visible, near infrared, and mid infrared for many years [5] addressing issues like control of optical path, high symmetry and matching of angles, pupil orientations, polarizations, spectrum, dispersion, etc. In the mid infrared [6] components availability is more difficult, however the foremost parameter of optical path control is easier to achieve due to $15 x$ longer wavelength. For JPLs future space missions such as TPF (D 4m) [7] and for the more recent space project PICTURE (a sounding rocket experiment scheduled for 2007) and other applications, we developped the visible nulling testbed at JPL. The experiment has gone through continuous technological and experimental progress over the years $[8,9,10]$. More progress and deeper nulls have occurred within the last year, which is the subject of the present paper.

\section{Nuller experimental description}

\subsection{The RoofTop setup and the new $W$ setup}

The visible nulling testbed at JPL is primarily a modified Mach Zender type interferometer, with single pupil input, described in many papers over the years, particularly in the form of the RSI (Rotational Shear) and field flip approach $[8,9,10]$ using rooftop (2 mirrors) until 2004. A benefit of rooftop setups is that OPD and shear can be adjusted independently. In early 2005 we built a W configuration (3 mirror nuller, with an additional reflection), see figure 1, with lower incidence angles to accommodate well a future deformable mirror for future projects and for tank operation. The old rooftop configuration and the current $\mathrm{W}$ configuration will soon be merged into yet another configuration (the Boomerang) for the rocket experiment, to merge both benefits.

The nuller is essentially an interferometer with two beam splitters and a set of mirrors on each side, defining two arms of high symmetry, connected to an input single mode fiber to a choice of light sources, and connected to an output single mode fiber to a choice of detectors, all of this with remote control and computer acquisition. The heart of the nuller is a pair of quality beam splitters with dielectric coatings and with very symmetric specifications. It is important to note that wavefront quality is not critical since the output fiber (core) acts as a single mode spatial filter. Geometrically the beam diameter was $20 \mathrm{~mm}$, angles of incidence $\sim 15 \mathrm{deg}$ everywhere, and each arm $\sim 25$ inches of path. In terms of polarization (not critical for white light) we had the capability to insert (zero deviation) polarizers at the input or at the output OAP levels or both. The nuller was also equipped with two shutters. To equalize OPDs, the adjustment was coarse (picomotor of range $25 \mathrm{~mm}$ ) to locate the white light fringe, then fine (piezzo PI stage P752 fed by a 100Volt generator) to explore and null the central fringe. Figure 1 shows elements for both setups.

\subsection{Sources}

We used laser and filtered white light (broadband) sources. For laser sources we used our old 650nm laser diode, then in 2004 a stronger laser diode module from Melles Griot emitting at $638 \mathrm{~nm}$ with a pigtailed power capable of $11 \mathrm{~mW}$ but tuned to $4 \mathrm{~mW}(3.75 \mathrm{~V}$, $80 \mathrm{~mA}$ ) for better stability. Lasers showed in fact linewidths of $\sim 1 \mathrm{~nm}$ ie $0.15 \%$ bandpass. The white light source used was of incandescent type equipped with filters centered at 


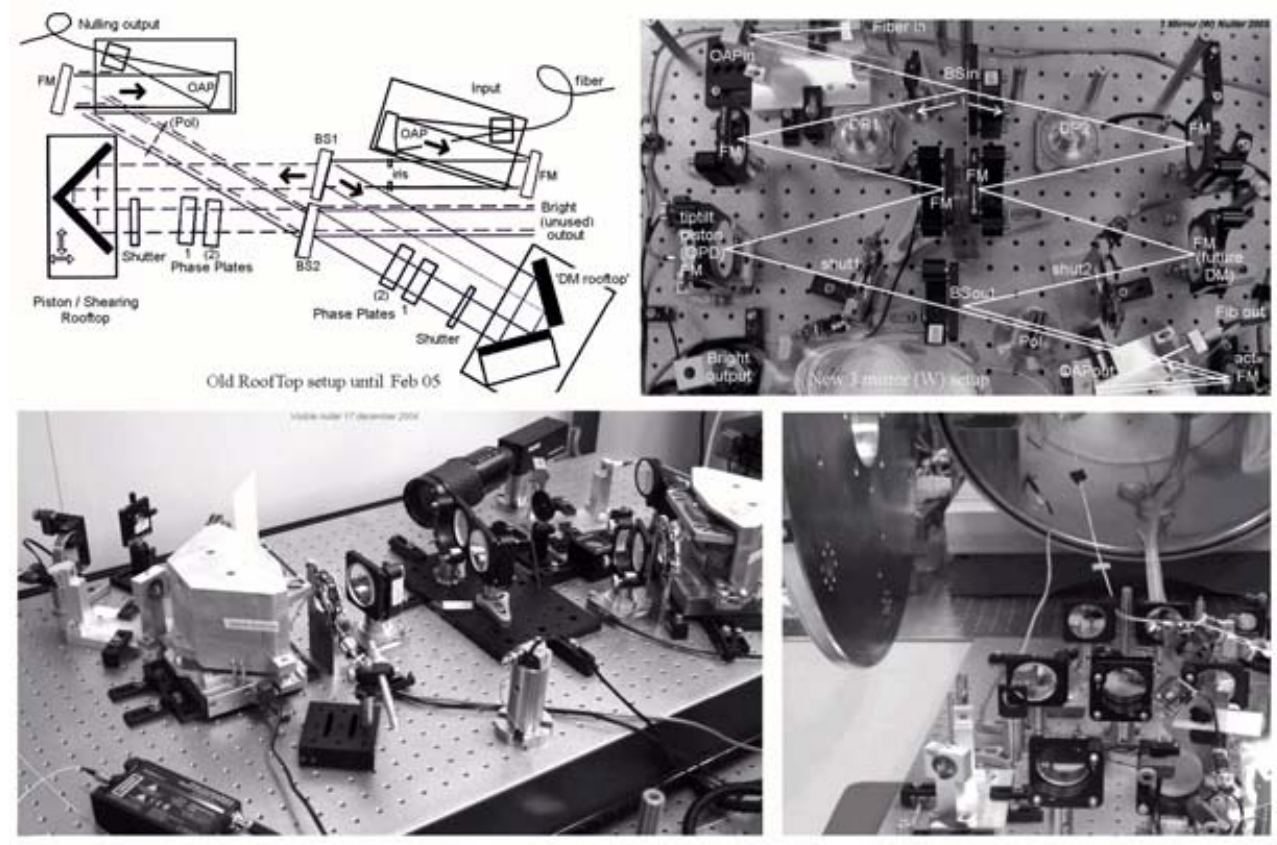

Figure 1. layout and photo of: the old RoofTop nuller (left), and new W nuller (right).

$650 \mathrm{~nm}$ and with relatively large bandpass of $5 \%$ and $12 \%$, and sometimes $20 \%$, verified by spectrometer (after the nuller).

\subsection{Detectors}

In order to see very low broadband flux typical of future starlight we had to use an Avalanche Photo Diode hooked up to a high speed counter. The APD detector module used was type SPCM AQR from Perkin Elmer with an advertised QE of $\sim 65 \%$ and noise of $\sim 30$ cps (counts per second). Due to high dynamic ranges encountered it was important to apply corrections, also to take precautions not over illuminate and burn the APD. The raw count was first noise corrected (dark current typically $\sim 50 \mathrm{cps}$ depending on situations), then for higher counts (more than $\approx 100 \mathrm{Kcps}$ ) we applied a correction due to the non linear behavior. For laser nulling we could use more traditional photodetectors such as model 2151 from New Focus for high $(\mathrm{KHz})$ speed, and the duochannel multimeter 2532C from Newport for convenient auto-ranging (largest dynamic range from miliWatts to sub nanoWatts). Usually no corrections were applied for these detectors as confirmed with neutral density filter ramp tests.

\subsection{Data processing}

The overall experiment was controlled with a PC under LabView giving the capability to read and control almost every instrument remotely. Typically a dataset would last several minutes and would contain the many features: intensity balance (i1-i2-i1-i2 switching), max intensity (top), manual surfing of the null (taking advantage of nanometric drifts we could do many passes to 'sense' the null), dark, midfringe. For broadband nulling we also recorded the +1 and -1 fringes. Nulling data was acquired over sampling rates of $25 \mathrm{~Hz}$ or $10 \mathrm{~Hz}$ with a N.I. DAQ under 16 bits. 


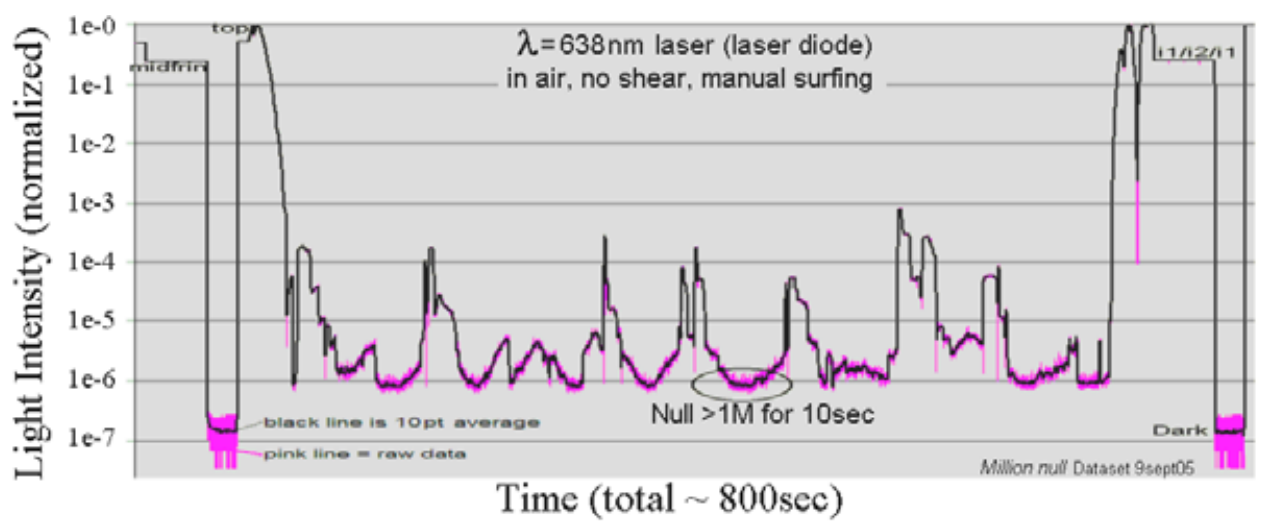

Figure 2. first 1 Million null under visible (638nm) laser light using a Mach Zender type nuller. The plot shows light intensity (log scale) versus time while OPD manual readjustements are done. The steady null is slightly better than $1 \mathrm{M}$ for 10 seconds. This results was obtained in air.

\section{Laser results and diagnosis}

Continuous progress and improvements over the years led to laser nulls in the $100 \mathrm{~K}$ range and broadband nulls in the $10 \mathrm{~K}$ ranges $[8,9,10]$. In spring 2005 we have reached $300 \mathrm{~K}$ laser null. Only recently, on June 10th 2005, we officially reached the milestone of 1 Million average null (ie 1e-6 rejection ratio, in this paper we use both notations equivalently) under monochromatic (laser) light, in air, tank closed, with a polarizer at the output. To this date we have reached slightly better (1.2 Million average raw null over many seconds, 1.4 Million corrected), see figure 2 . To our knowledge this is the best null ever in the visible.

A note about the terminology of transient nulls (single datapoints): transient nulls have been quoted many times in past papers and can be very deep, but they are merely a consequence of low flux statistical noise. In this paper and for future nulling papers we declare that transient nulls are not scientifically meaningful of the steady null, and should be phased out, we recommend doing an average over at sufficient number of consecutive points (here 10 points ie $1 \mathrm{sec}$ ) to claim a null result.

A variety of conditions had to be met and meticulously optimized to reach very deep nulls. It takes only one to degrade an otherwise deep null. The top two factors that were easy to access were OPD noise and power imbalance. OPD noise was easily deduced from midfringe (PSD/Fourier) analysis, recording light signal at mid interference using a fast rate $(\mathrm{KHz})$ photodetector. The (quadratic) rule of thumb was that $0.1 \mathrm{~nm}$ RMS OPD limits the null to $\sim 2.5 \mathrm{e}-7$ solely from this contribution (a $0.1 \mathrm{~nm}$ side shift causes 1e-6). For the power imbalance (mismatch) the light trace i1/i2/i1/i2 was recorded while the shutters were clicked alternatively. Here the rule of thumb was that $0.15 \%$ intensity mismatch limits null to $2.8 \mathrm{e}-7$ solely from this contribution. To fine tune intensities to the $0.1 \%$ or better level we used a fine tip tilt control (picomotor) within or outside the nuller (before the output OAP). The latter operation mitigated the Airy spot positions on the core to intercept equal amounts of light from each arm. A technique of a thin wire occultator is now tested to give possibly better stability and resolution, reminding us of the past when we used a venitian blind technique in our Rotational Shear Interferometer [9]. Analyzing our $1 \mathrm{M}$ null we found $0.1 \mathrm{~nm}$ RMS for the OPD noise and $0.15 \%$ for the power mismatch, see figure 3 . Therefore the sum would be $\sim 5 \mathrm{e}-7$ versus $7 \mathrm{e}-7$ measured, so there was more contributor(s) in the null depth. If these were identified and reduced the current experiment could possibly get us to $1 \mathrm{e}-7$ nulls. 

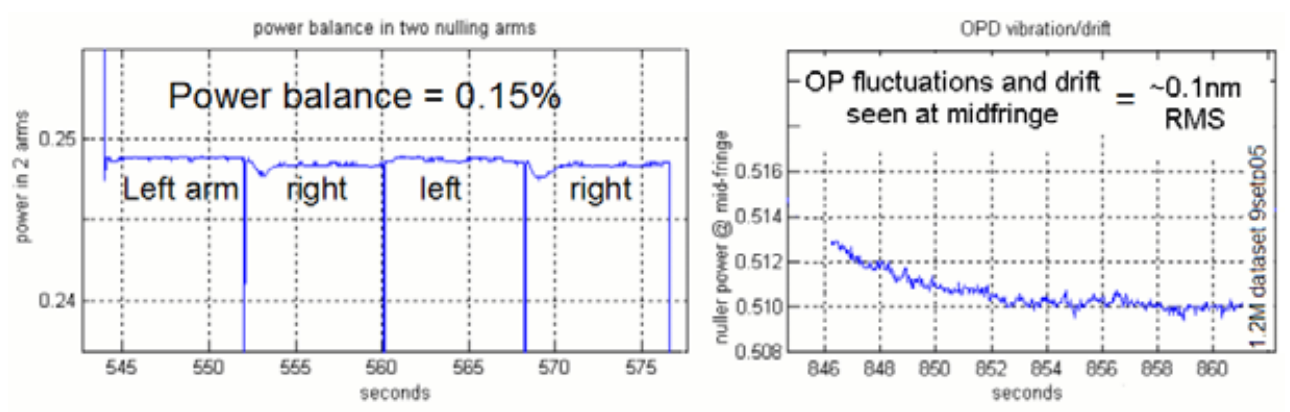

Figure 3. diagnosis of the 1 million null in terms of intensity mismatch and OPD noise.

A third factor under recent investigation is angular/polarization mismatch This parameter is not easy to sense but we have ideas such as high precision polarimetry or precise alignment. From a geometric point of view we need to align the angle of incidence on optics ( $\sim 15 \mathrm{deg})$ to the arcminute level, as well as nuller planarity and wedge orientations, also make sure about mirror coatings and pairwise matching of coatings for the beam splitters. We are currently rebuilding the nuller under an autocollimator to the arcminute level. If we find a null better than a million then it is the nuller that was not well aligned.

\section{Environment}

Environmental conditions are often under estimated and have not been described enough in previous papers, but play a major role in very deep (more than $100 \mathrm{~K}$ ) nulling experiments. Many conditions must be perfect or optimized simultaneously, with the foremost condition of zero optical path difference between the two arms. A rule is that any ambient condition or device generating noise and vibrations will have to be stopped, removed or located far away from the nuller bench or enclosure. This was easier said than done: typically air conditioning, always present in American buildings, would cause significant noise at the subnanometer level. Computer fans would cause noise acoustically and through cables coupling, with the constraint that is was not possible to place the computer very far away to the nuller and enclosure. In the nuller itself a major disturbance was air turbulence: devices thought to be quiet such a CCD camera or a shutter were found to generate a tiny amount of heat sufficient to cause slow micro turbulence and fluctuations around the paths at the $1 \mathrm{M}$ level (see dips in fig. 3 left). We plan to install a zero heat shutter. A non obvious and in fact paradoxical disturbance was that the piston piezzo itself, normally used to zero the OPD, would show a lot of creep at the subnm level. Definitely the internal pzt servo, normally sought to combat piezzo creep and hysteresis, would add servo noise, we therefore never used this servo when deep nulling, obligeing to manually track and surf the null, hence the irregular aspect of the null bottom on the plots shown in this paper. For tip tilt and OPD control we used the popular picomotors, aka the piezzo that turns a screw, from New Focus (for some years now). The fact that they have a small step size $(10-20 \mathrm{~nm})$, large range, and can hold a position without power (heat) or vibrations, is much an advantage compared to some drawbacks such as step non repeatability or the need of high voltage pulse generators. Following tests we have never evidenced any picomotor noise or drifts between positions (between tics), an essential ingredient to deep nulling. The nulling bench was resting on a couple of elastic sorbothane pucks which were confirmed to dramatically cutoff frequencies in the $\mathrm{Hz}-\mathrm{KHz}$ range, also the main optical table was on traditional floatation. At 


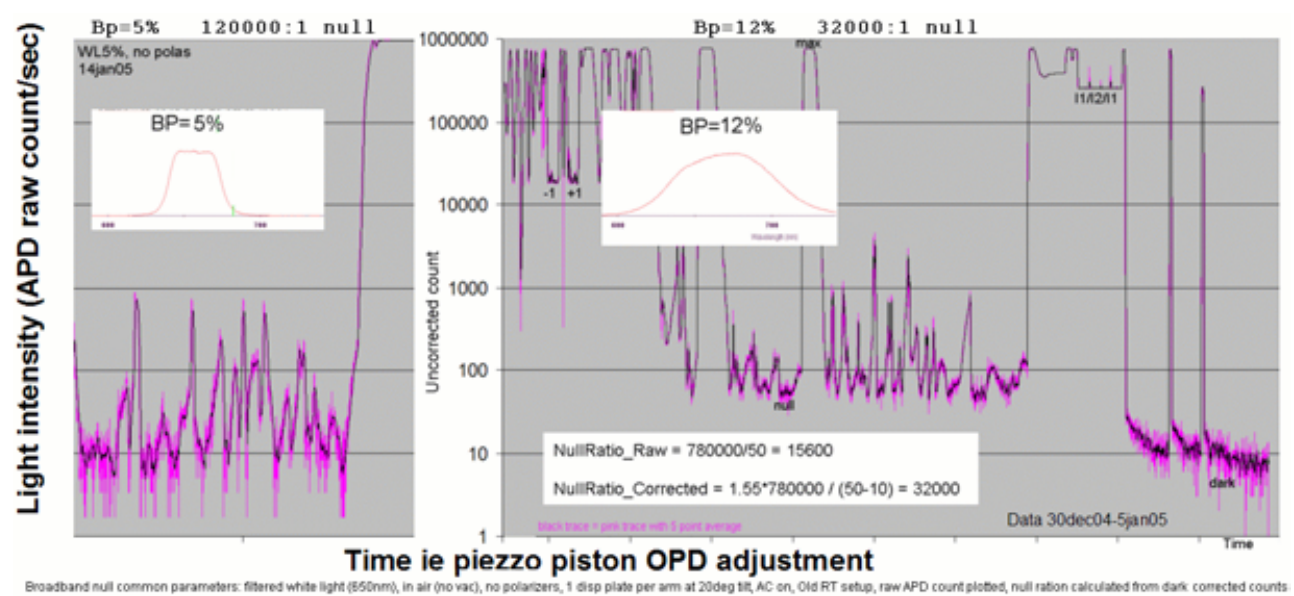

Figure 4. broadband nulls at $650 \mathrm{~nm}$ of about 120000 and 32000 with $\mathrm{BP}=5 \%$ and $12 \%$.

least 30 minutes of settling time was needed to start any deep null. For the old rooftop setup $[8,9,10]$ the nuller was enclosed in the aquarium, a $2 \times 1 \times 1 m$ box of plexiglass with inner foam panels. The aquarium has not been cutting enough acoustics to reach $1 \mathrm{M}$ nulls, especially air conditioning noise and vibrations. For the new setup (W) the nuller bench was inserted in a small vacuum chamber in metal. By placing the fringe-o-phone (an apparatus with a headphone connected to a photodiode with amplifier built to diagnose opto mechanical instability) to the output fiber, during a laser null sitting near mid fringe, ambient noises such as conversations were heard into the headphones showing that acoustic noise was directly coupling into the OPD. We deduced that any enclosure (plexi or metal) drastically reduced micro air currents in the arms but had almost no effect on acoustic attenuation. The ultimate remedy for this would be vacuum. When we started vaccum tests we verified, doing midfringe analysis and using the fringe-o-phone, that the nuller was very quiet, nevertheless we saw drifts (plate relaxation, thermals, etc). So far we have always seen better results operating in enclosed air, with door closed, in evenings and quiet hours, rather than operating under vacuum. We have obtained the $1 \mathrm{M}$ null (fig. 2) in air, with tank door closed.

\section{Broadband nulling}

Whereas nulling interferometry is easiest for monochromatic light (laser), scientific and astronomical interest lies only in broadband (star light) capability. Much of an improvement occured in fall 2004 when we installed two small Off Axis Parabola (OAP) mirrors (see fig. 1), replacing the doublet lens collimators, to correct longitudinal chromatic aberration. Each OAP was polished to a figure of $\lambda / 20 \mathrm{PV}$ and mounted in a near monolithic aluminum mount design for stability and compactness. The nuller was equipped with tilted glass plates (dispertion plates) as described in the past $[8,9,10]$ to create varying glass effective thickness between the right and the left arm. For broadband visible performance the effective thickness difference was calculated to be 20 micron, with $\sim 29$ micron of extra air in the other arm to compensate. Empirically we adjusted the angles of the plates, using rotary picomotors, to symmetrize the +1 and -1 fringe on each side of the null (symmetry of the fringe packet). When exploring the rotation fringe space, we found deeper nulls and higher order nulls. 


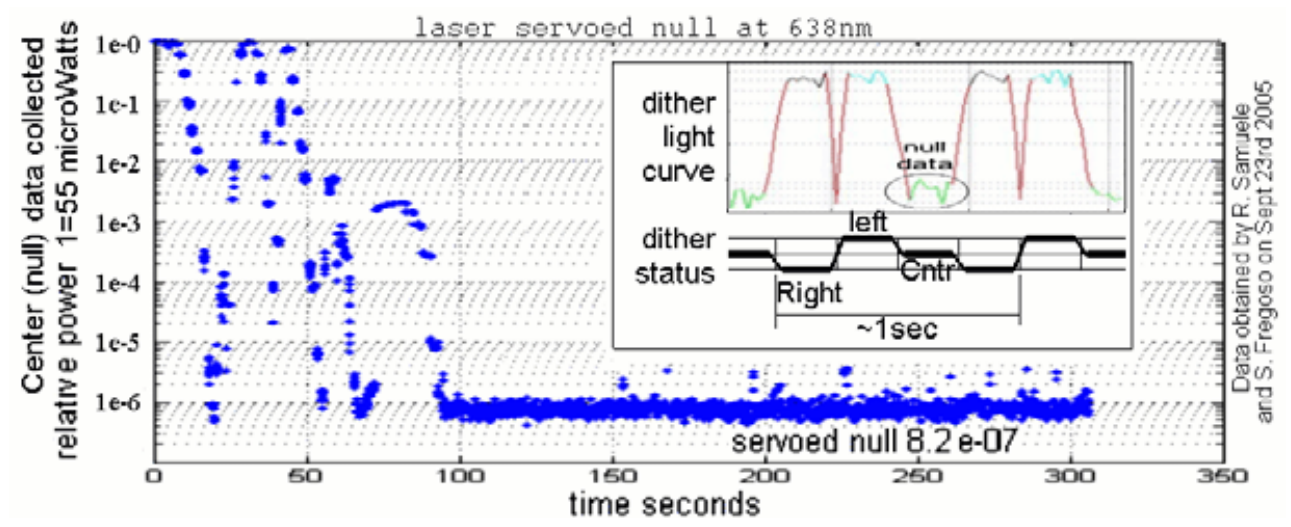

Figure 5. servo ie tracked laser null of $1.2 \mathrm{M}$ ie $8.2 \mathrm{e}-7$ over 200 s (average, corrected).

Using properly tuned plates in the old (rooftop) setup, in air, without polarizers, with filtered red light at $650 \mathrm{~nm}$, we obtained a null of 120000 for $5 \%$ bandpass, and 32000 for $12 \%$ bandpass, see figure 4 . Unfortunately we have not attempted $18-20 \%$ so a direct comparison with our past result [10] is not possible. Note that in the left of the $12 \%$ plot fringes +1 and -1 were well equalized, also note a large $(2 \mathrm{x})$ difference between raw and corrected due to low flux. We have experimented only one glass type per arm, but have plans for 2006 to install two glasses per arm to augment bandwidth. With two glasses the procedure will be much more complex and the difference of effective thickness would theoretically be $\sim 400$ microns to give $10 \mathrm{M}$ over $20 \%$.

\section{Servoed null}

A recent (fall 2005) result is that we were able to track a 1 Million laser null, see figure 5, which is a significant improvement over our last tracked result of a little bit better than 10000 uncorrected obtained in 2000 [10]. We implemented a servo technique based simply on the signal itself, using the weak flux around null, as opposed to other schemes typically using heterodyne laser metrology to monitor paths (in fact we implemented this a few years ago), or a quadrature output [11]. We applied a small OPD dither cycle around the null with 3 plateau positions called left, center (null) and right and monitored the light level at these 3 points. From any difference between the right and left levels we could easily tell any drift away from the null. Under LabView, with the proper conversion factor, we fed back the positive or negative error signal into the piston piezzo to bring the null back. In terms of servo parameters we used a relatively slow cycle rate of $1 \mathrm{~Hz}$ given the low flux (55 microWatts on top of the fringe, 55picoWatts at null), we had a duty cycle of $\sim 25 \%$ ( 8 null points out of 33 ), and a dither excursion of $\sim 10 \mathrm{~nm}$. The closed loop operation added a minimal amount of noise and did not declassify the original 1 Million null. We finally tested correct function of the closed loop by applying small OPD disturbances and verifying that the null would (slowly) come back despite of the very low flux captured at the left and right points.

\section{Conclusion}

Using a modified Mach Zender interferometer as our nuller tesbed we have developed technologies and experimental progress to reach very deep nulls needed for future NASA planet detecting missions such as TPF, or PICTURE (an upcoming sounding rocket 
experiment for launch in 2007), and more generally for the American and European planet finding communities. Under visible laser light $(638 \mathrm{~nm})$ we have reached the 1 Million null ratio milestone (ie 1e-6 rejection ratio) on June 10th 2005, in fact to this date we have obtained a slightly better null of 1.2 Million (average raw null over many seconds). To our knowledge this is the first time that a visible Mach Zender nuller has surpassed the Million. To reach this type of null we have worked on many conditions such as optical path difference stability, optical alignment, symmetry in the two arms, mechanical stability and vibration isolation. In terms of broadband nulling, of more astronomical interest, under unpolarized filtered white light at $650 \mathrm{~nm}$, and using one glass plate in each arm, we obtained a null of 120000 with a bandpass of $5 \%$, and 32000 with a larger bandpass of $12 \%$. Moreover we report a latest result of closed loop nulling: we have been able to track the 1 Million laser null, with a slow bandwith and with minor noise degradation. We are glad to report that all four results were obtained in air, without pumping vacuum yet. With a 1e-6 rejection ratio we are only 10 times away from TPFs goal of 1e-10, indeed using a combination of a fiber array and deformable mirror a null

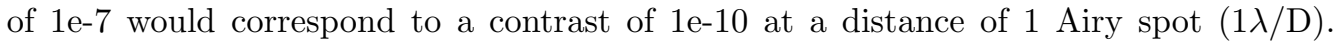
Future plans are to add shear, progress on deep laser nulling (goal of $2 \mathrm{M}$ soon, then eventually 10M) as well as an effort on broadband nulling up to $20 \%$ bandpass (goal of $1 \mathrm{M}$ at $5 \%$ soon), which likely needs 2 glasses per arm. In parallel to these efforts we are starting to introduce certain devices for upcoming projects such as a deformable mirror with an associated array of lenslets and single mode fibers [7], a downstream addition of a calibration system, and more remote control for a more end to end system.

\section{Acknowledgments}

We thank TPF and PICTURE for the support of this work. We thank S.F. Fregoso for help with data acquisition and LabView programmation. This work was performed at the Jet Propulsion Laboratory of the California Institute of Technology, under contract with the National Aeronautics and Space Administration.

\section{References used}

[1] M. Mayor, D. Queloz, Jupiter-mass companion to a solar-type star, Nature 378, 355 (1995)

[2] A. Burrows, A theoretical look at the direct detection of giant planets outside the solar system, Nature, vol. 433, 261, (2005)

[3] Jean Schneiders exo planet list, daily updated, www.obspm.fr

[4] R. Bracewell, Detecting nonsolar planets by spinning infrared interferometer. Nature 274, $780-781$ (1978)

[5] E. Serabyn, Nulling Interferomtry Progress, Proc SPIE, 4838 (2003)

[6] S.R. Martin, R.O. Gappinger, F.M. Loya, B.M. Menesson, et al., A mid infrared nuller for TPF: design, progress, results, proc SPIE, vol 5170, (2003)

[7] B.M. Levine, M. Shao et al, Visible nulling coronograph architecture definition and tech development, this proc

[8] Serabyn, E., Wallace, J. K., Hardy, G. J., Schmidtlin, E. G. H., Nguyen, H. Deep nulling of visible laser light, Appl. Opt. 38, 7128-7132 (1999)

[9] Serabyn, E., Wallace, J. K., Nguyen, H. T., Schmiditlin, E. G. H., Hardy, G. J. in Working on the Fringe: Optical and IR Interferometry from Ground and Space (eds Unwin, S. and Stachnik, R.) 437-442 (1990)

[10] K. Wallace, G. Hardy, E. Serabyn, Deep and stable interferometric nulling of broadband light, Nature, August 2000, 406, 700-702 (2000)

[11] E. Serabyn, Nanometer-level path-length control scheme for nulling interferometry. Appl. Opt. 38, 4213-4216 (1999) 